\title{
An isomorphism theorem for Dragilev spaces
}

\author{
By \\ MEFHARET KoCATEPE*)
}

Necessary and sufficient conditions for an $L_{f}(a, 1)$-space to be isomorphic to some $L_{g}(b, \infty)$-space have been found.

Introduction. In [2] Dragilev has claimed that if $r, s \in\{+\infty, 1,0,-1\}$ and $r \neq s$, then for any two rapidly increasing Dragilev functions $f$ and $g$ and for any two sequences $a=\left(a_{i}\right)$ and $b=\left(b_{i}\right)$, the spaces $L_{f}(a, r)$ and $L_{g}(b, s)$ cannot be isomorphic. In [4] and [5], by means of examples it was shown that this is not true for $(r, s)=(1,+\infty)$ and $(r, s)=(-1,0)$.

In this note we characterize those $L_{f}(a, 1)$ spaces which are isomorphic to $L_{g}(b, \infty)$ spaces. The characterization is given in terms of the functor Ext and a condition which is obtained by comparing the diametral dimensions of the two spaces.

Preliminaries. Let $f$ be an odd, increasing, logarithmically convex function (i.e. $\varphi(x)=\log f\left(e^{x}\right)$ is convex). Throughout this paper such a function will be called a Dragilev function. Let $a=\left(a_{i}\right)$ be a strictly increasing sequence of positive numbers with $\lim a_{i}=+\infty$ and $\left(r_{k}\right)$ a strictly increasing sequence of real numbers with $\lim r_{k}=r$ where $-\infty<r \leqq+\infty$. The Dragilev space $L_{f}(a, r)$ is defined as the Köthe space $\lambda(A)$ generated by the matrix $A=\left(a_{i}^{k}\right), a_{i}^{k}=\exp f\left(r_{k} a_{i}\right)$ (see [2]).

By logarithmic convexity of $f$ we have that for every $a>1, \tau(a)=\lim _{x \rightarrow+\infty}(f(a x) / f(x))$ exists.

Moreover either (i) $\tau(a)<+\infty$ for all $a>1$, or (ii) $\tau(a)=+\infty$ for all $a>1$. $f$ is called slowly increasing in the first case, rapidly increasing in the second case. It is well-known that $L_{f}(a, r)$ is isomorphic to a power series space if and only if $f$ is slowly increasing. In this paper we shall consider only rapidly increasing Dragilev functions.

In [7] several properties of functor $\operatorname{Ext}(E, F)=\operatorname{Ext}^{1}(E, F)$ for two Fréchet spaces $E$ and $F$ were given. It was shown in [1] that $\operatorname{Ext}\left(L_{g}(b, \infty), L_{g}(b, \infty)\right)=0$ and in [3] that $\operatorname{Ext}\left(L_{f}(a, 1), L_{f}(a, 1)\right)=0$ if and only if there is a number $c>1$ such that the set of limit points of the set $\left\{a_{j} / a_{i}: i, j \in \mathbb{N}\right\}$ is contained in $[0,1] \cup[c,+\infty]$.

Results. We first give a necessary condition for $L_{f}(a, 1)$ to be isomorphic to some $L_{g}(b, \infty)$.

*) This research was supported by the Scientific and Technical Research Council of Turkey. 
Proposition 1. If $L_{f}(a, 1)$ is isomorphic to some $L_{g}(b, \infty)$, then there is a strictly increasing sequence $\left(r_{k}\right)$ of positive numbers with $\lim r_{k}=1$ and there is a strictly increasing function $p: \mathbb{N} \rightarrow \mathbb{N}$ such that

$$
\frac{f\left(r_{k+1} a_{i}\right)}{f\left(r_{k} a_{i}\right)} \leqq \frac{f\left(r_{k+2} a_{i}\right)}{f\left(r_{k+1} a_{i}\right)}, \quad k \in \mathbb{N}, i \geqq p(k) .
$$

The proof of this proposition is essentially given in [4] (Proposition 1). The only difference is that we choose $r_{k}$ slightly larger than the one chosen in [4], so that the inequality above holds for all large $i$ (depending on $k$ ).

Before our next proposition we observe the following.

$\mathrm{Re} \mathrm{m}$ a $\mathrm{r}$. If a positive sequence $\left(r_{k}\right)$ strictly increases to 1 , then there is a $k_{0} \in \mathbb{N}$ such that

$$
\frac{r_{k+1}}{r_{k}} \leqq \frac{r_{2}}{r_{1}}, \quad k \geqq k_{0}
$$

This follows from $\lim r_{k+1} / r_{k}=1$ and $r_{2} / r_{1}>1$.

Proposition 2. Suppose $\inf \left(a_{i+1} / a_{i}\right)=a>1$ and there is a strictly increasing positive sequence $\left(r_{k}\right)$ with $\lim r_{k}=1$ and there is a strictly incresing function $p: \mathbb{N} \rightarrow \mathbb{N}$ such that

$$
\frac{f\left(r_{k+1} a_{i}\right)}{f\left(r_{k} a_{i}\right)} \leqq \frac{f\left(r_{k+2} a_{i}\right)}{f\left(r_{k+1} a_{i}\right)}, \quad k \in \mathbb{N}, i \geqq p(k) .
$$

Then $L_{f}(a, 1)$ is isomorphic to some $L_{g}(b, \infty)$.

P roof. By the previous remark, by passing to a subsequence of $\left(r_{k}\right)$ if necessary we may assume that

$$
\frac{r_{k+1}}{r_{k}} \leqq \frac{r_{2}}{r_{1}}, \quad k \in \mathbb{N}
$$

Since $\inf \left(a_{i+1} / a_{i}\right)=a>1$, there is a $k_{0}$ such that $r_{k_{0}}>1 / a$. Again by passing to a subsequence of $\left(r_{k}\right)$ if necessary we may assume that $r_{1}>1 / a$, that is

$$
r_{k} a_{i}<a_{i} \leqq \frac{a_{i+1}}{a}<r_{1} a_{i+1}, \quad i, k \in \mathbb{N} .
$$

Then by using logarithmic convexity of $f$, (1) and (2) for $i, k \in \mathbb{N}$ we have

$$
\begin{aligned}
\log \frac{f\left(r_{k+1} a_{i}\right)}{f\left(r_{k} a_{i}\right)} & =\frac{\varphi\left(\log \left(r_{k+1} a_{i}\right)\right)-\varphi\left(\log \left(r_{k} a_{i}\right)\right)}{\log r_{k+1}-\log r_{k}}\left(\log r_{k+1}-\log r_{k}\right) \\
& \leqq \frac{\varphi\left(\log \left(r_{2} a_{i+1}\right)\right)-\varphi\left(\log \left(r_{1} a_{i+1}\right)\right)}{\log r_{2}-\log r_{1}}\left(\log r_{k+1}-\log r_{k}\right) \\
& =\log \frac{f\left(r_{2} a_{i+1}\right)}{f\left(r_{1} a_{i+1}\right)} \frac{\log r_{k+1}-\log r_{k}}{\log r_{2}-\log r_{1}} \leqq \log \frac{f\left(r_{2} a_{i+1}\right)}{f\left(r_{1} a_{i+1}\right)},
\end{aligned}
$$


where $\varphi(x)=\log f\left(e^{x}\right)$ which is convex. That is, we have

$$
\frac{f\left(r_{k+1} a_{i}\right)}{f\left(r_{k} a_{i}\right)} \leqq \frac{f\left(r_{2} a_{i+1}\right)}{f\left(r_{1} a_{i+1}\right)}, \quad i, k \in \mathbb{N} .
$$

Now let $i_{0}=\min \{p(k): k \geqq 2\}=p(2)$ and for $i \geqq i_{0}$, define $k(i)=\max \{k: p(k) \leqq i\}$. Then

$$
\begin{gathered}
2=k\left(i_{0}\right) \leqq k(i) \leqq k(i+1) \quad \text { for } \quad i \geqq i_{0}, \quad \lim _{i \rightarrow \infty} k(i)=+\infty \\
\text { and } p(k(i)) \leqq i \text { for } i \geqq i_{0} .
\end{gathered}
$$

Rewriting the hypothesis we have

$$
\frac{f\left(r_{k+1} a_{i}\right)}{f\left(r_{k} a_{i}\right)} \leqq \frac{f\left(r_{k+2} a_{i}\right)}{f\left(r_{k+1} a_{i}\right)}, \quad i \geqq i_{0}, \quad k(i) \geqq k .
$$

Next we choose $s>1$ and fix it, and define $s_{k}=s^{k}$. Then we define a sequence $b=\left(b_{i}\right)$, $i \geqq i_{0}$ as follows: $b_{i_{0}}=1$ and $b_{i+1}$ is inductively defined by

$$
\log \frac{b_{i+1}}{s^{k(i)-1} b_{i}}=\log s \frac{\log f\left(r_{1} a_{i+1}\right)-\log f\left(r_{k(i)} a_{i}\right)}{\log f\left(r_{k(i)} a_{i}\right)-\log f\left(r_{k(i)-1} a_{i}\right)} .
$$

By (2), the right hand side is positive and so $s^{k(i)-1} b_{i}<b_{i+1}$, that is $s_{k(i)} b_{i}<s_{1} b_{i+1}$.

Also by (3) we have

$$
\frac{\log f\left(r_{1} a_{i+1}\right)-\log f\left(r_{k(i)} a_{i}\right)}{\log f\left(r_{2} a_{i+1}\right)-\log f\left(r_{1} a_{i+1}\right)} \log s \leqq \frac{\log f\left(r_{1} a_{i+1}\right)-\log f\left(r_{k(i)} a_{i}\right)}{\log f\left(r_{k(i)} a_{i}\right)-\log f\left(r_{k(i)-1} a_{i}\right)} \log s,
$$

that is (by using definition of $b_{i+1}$ )

$$
\begin{aligned}
\frac{\log f\left(r_{1} a_{i+1}\right)-\log f\left(r_{k(i)} a_{i}\right)}{\log f\left(r_{2} a_{i+1}\right)-\log f\left(r_{1} a_{i+1}\right)} \log s & \leqq \log \frac{s_{1} b_{i+1}}{s_{k(i)} b_{i}} \\
& \leqq \frac{\log f\left(r_{1} a_{i+1}\right)-\log f\left(r_{k(i)} a_{i}\right)}{\log f\left(r_{k(i)} a_{i}\right)-\log f\left(r_{k(i)-1} a_{i}\right)} \log s
\end{aligned}
$$

or since $\log \left(s_{k} b_{i}\right)-\log \left(s_{k-1} b_{i}\right)=\log s$, for $i \geqq i_{0}$ we equivalently have

$$
\begin{aligned}
\frac{\log f\left(r_{k(i)} a_{i}\right)-\log f\left(r_{k(i)-1} a_{i}\right)}{\log \left(s_{k(i)} b_{i}\right)-\log \left(s_{k(i)-1} b_{i}\right)} & \leqq \frac{\log f\left(r_{1} a_{i+1}\right)-\log f\left(r_{k(i)} a_{i}\right)}{\log \left(s_{1} b_{i+1}\right)-\log \left(s_{k(i)} b_{i}\right)} \\
& \leqq \frac{\log f\left(r_{2} a_{i+1}\right)-\log f\left(r_{1} a_{i+1}\right)}{\log \left(s_{2} b_{i+1}\right)-\log \left(s_{1} b_{i+1}\right)} .
\end{aligned}
$$

Now we choose $i_{1} \geqq i_{0}$ such that

$$
\log f\left(r_{2} a_{i_{1}}\right)-\log f\left(r_{1} a_{i_{1}}\right) \geqq \log s .
$$

Then we define $\psi\left(\log \left(s_{k} b_{i}\right)\right)=\log f\left(r_{k} a_{i}\right), i \geqq i_{1}, k \leqq k(i)$, and $P_{k, i}=\left(\log \left(s_{k} b_{i}\right), \psi\left(\log \left(s_{k} b_{i}\right)\right)\right)$ and join the points

$$
P_{1, i_{1}} \rightarrow \cdots \rightarrow P_{k\left(i_{1}\right), i_{1}} \rightarrow \cdots \rightarrow P_{1, i} \rightarrow P_{2, i} \rightarrow \cdots \rightarrow P_{k(i), i} \rightarrow P_{1, i+1} \rightarrow P_{2, i+1} \cdots
$$


by line segments. This way $\psi$ is defined for all $x \geqq \log \left(s_{1} b_{i_{1}}\right)$. For $x \leqq \log \left(s_{1} b_{i_{1}}\right)$, we define $\psi(x)=\log f\left(r_{1} a_{i_{1}}\right)-\log \left(s_{1} b_{i_{1}}\right)+x$. It is clear that $\psi$ is increasing. By (4) we have that $\psi$ is convex within the $i$-th block and from (5) it follows that the slope of $\psi$ increases when we pass from the $i$-th to the $(i+1)$-st block. Finally $i_{1}$ was chosen in such a way that the slope of $\psi$ from $(0, \psi(0))$ to $P_{1, i_{1}}$ is smaller than the slope from $P_{1, i_{1}}$ to $P_{2, i_{1}}$.

Now we define

$$
g(x)=\left\{\begin{array}{lc}
\frac{f\left(r_{1} a_{i_{1}}\right)}{s_{1} b_{i_{1}}} x, & 0 \leqq x \leqq s_{1} b_{i_{1}} \\
e^{\psi(\log x)}, & s_{1} b_{i_{1}} \leqq x \\
-g(-x), & x \leqq 0 .
\end{array}\right.
$$

Then $g$ is an increasing, odd function with $\log g\left(e^{x}\right)=\psi(x)$, and so $g$ is logarithmically convex.

Finally for $i \geqq i_{1}$ and $k \leqq k(i)$, that is for $i \geqq \max \left\{p(k), i_{1}\right\}$ we have $f\left(r_{i k} a_{i}\right)=g\left(s_{k} b_{i}\right)$, which shows that $L_{f}(a, 1)$ is isomorphic to $L_{g}(b, \infty)$.

Next proposition extends Proposition 2.

Proposition 3. Suppose $\operatorname{Ext}\left(L_{f}(a, 1), L_{f}(a, 1)\right)=0$ and there is a strictly increasing sequence $\left(r_{k}\right)$ with $\lim r_{k}=1$ and a strictly increasing function $p: \mathbb{N} \rightarrow \mathbb{N}$ such that

$$
\frac{f\left(r_{k+1} a_{i}\right)}{f\left(r_{k} a_{i}\right)} \leqq \frac{f\left(r_{k+2} a_{i}\right)}{f\left(r_{k+1} a_{i}\right)}, \quad k \in \mathbb{N}, i \geqq p(k) .
$$

Then $L_{f}(a, 1)$ is isomorphic to some $L_{g}(b, \infty)$.

Proof. By [6], $\operatorname{Ext}\left(L_{f}(a, 1), L_{f}(a, 1)\right)=0$ if and only if the pair $\left(L_{f}(a, 1), L_{f}(a, 1)\right)$ satisfies condition $\left(S_{2}^{*}\right)$, and it was shown in [3]. (p. 37 and p. 29) that this happens if and only if there is a number $c>1$ such that the set of limit points of $\left\{a_{j} / a_{i}: i, j \in \mathbb{N}\right\}$ is contained in $[0,1] \cup[c,+\infty]$. Let $a=(1+c) / 2>1$.

We set $i_{1}=1$ and choose $i_{2}$ as the smallest index $n$ such that $a_{n} / a_{i_{1}} \geqq a$, then we choose $i_{3}$ as the smallest index $n$ such that $a_{n} / a_{i_{2}} \geqq a$. We continue this way and choose a strictly increasing sequence $\left(i_{n}\right)$ of indices such that

$$
\frac{a_{i_{n+1}}}{a_{i_{n}}} \geqq a, \quad \frac{a_{i_{n+1}}-1}{a_{i_{n}}}<a, \quad n \in \mathbb{N} .
$$

Let $M=\left\{n: i_{n}+1<i_{n+1}\right\}$.

If $M$ is a finite set, then there is an $n_{0} \in \mathbb{N}$ such that for $n \geqq n_{0}, i_{n+1}=i_{n}+1$ and so for some $m_{0}, i_{n_{0}+n}=m_{0}+n$ for $n \geqq 1$. Hence

$$
\frac{a_{m_{0}+n+1}}{a_{m_{0}+n}}=\frac{a_{i_{n_{0}+n+1}}}{a_{i_{n_{0}+n}}} \geqq a, \quad n \geqq 1,
$$


and by Proposition 2, $L_{f}\left(\left(a_{i}\right)_{i>m_{0}}, 1\right)$ is isomorphic to some $L_{g}\left(\left(b_{i}\right)_{i>m_{0}}, \infty\right)$ and so $L_{f}(a, 1)$ is isomorphic to $L_{g}(b, \infty)$.

If $M$ is an infinite set, it follows from $a_{i_{n+1}-1} / a_{i_{n}}<a$ and from the property of $c$ that 1 is the only limit point of the bounded set

$$
\left\{\frac{a_{i_{n+1}-1}}{a_{i_{n}}}: n \in M\right\},
$$

and so $\lim _{n \in M}\left(a_{i_{n+1}-1} / a_{i_{n}}\right)=1$. Since $a_{i_{n+1}} / a_{i_{n}} \geqq a$, by Proposition $2, L_{f}\left(\left(a_{i_{n}}\right), 1\right)$ is isomorphic to some $L_{g}\left(\left(b_{i_{n}}\right), \infty\right)$ with $f\left(r_{k} a_{i_{n}}\right)=g\left(s_{k} b_{i_{n}}\right)$ for $n \geqq n_{k}$. For $n \in M$ and $i_{n}<i<i_{n+1}$ we define $b_{i}$ in such a way that $b_{i_{n}}<b_{i}<b_{j}<b_{i_{n+1}}$ if $i_{n}<i<j<i_{n+1}$ and $\lim _{n \in M}\left(b_{i_{n+1}-1} / b_{i_{n}}\right)=1$.

Now given $k$ we find $n_{0}$ such that

$$
\frac{a_{i_{n+1}}-1}{a_{i_{n}}}<\frac{r_{k+1}}{r_{k}}, \quad \frac{b_{i_{n+1}-1}}{b_{i_{n}}}<\frac{s_{k+1}}{s_{k}}, \quad n \geqq n_{0} .
$$

If $i_{n}<i<i_{n+1}$ for $n \geqq n_{0}$, then

$$
\frac{a_{i}}{a_{i_{n}}}<\frac{r_{k+1}}{r_{k}}, \quad \frac{b_{i}}{b_{i_{n}}}<\frac{s_{k+1}}{s_{k}}
$$

and so for $n \geqq \max \left\{n_{0}, n_{k+1}\right\}$ and $i_{n}<i<i_{n+1}$ we have

$$
\begin{aligned}
& f\left(r_{k} a_{i}\right) \leqq f\left(r_{k+1} a_{i_{n}}\right)=g\left(s_{k+1} b_{i_{n}}\right) \leqq g\left(s_{k+1} b_{i}\right), \\
& g\left(s_{k} b_{i}\right) \leqq g\left(s_{k+1} b_{i_{n}}\right)=f\left(r_{k+1} a_{i_{n}}\right) \leqq f\left(r_{k+1} a_{i}\right) .
\end{aligned}
$$

So $L_{f}(a, 1)$ is isomorphic to $L_{g}(b, \infty)$.

Now we combine our propositions and the fact that $\operatorname{Ext}\left(L_{f}(a, 1), L_{f}(a, 1)\right)=0$ is a necessary condition for $L_{f}(a, 1)$ to be isomorphic to some $L_{g}(b, \infty)$ in the following theorem.

Theorem. Let $f$ be a rapidly increasing Dragilev function. Then $L_{f}(a, 1)$ is isomorphic to some $L_{g}(b, \infty)$ if and only if the following conditions are satisfied:

(i) $\operatorname{Ext}\left(L_{f}(a, 1), L_{f}(a, 1)\right)=0$,

(ii) There is a strictly increasing sequence $\left(r_{k}\right)$ with $\lim r_{k}=1$ and a strictly increasing function $p: \mathbb{N} \rightarrow \mathbb{N}$ such that

$$
\frac{f\left(r_{k+1} a_{i}\right)}{f\left(r_{k} a_{i}\right)} \leqq \frac{f\left(r_{k+2} a_{i}\right)}{f\left(r_{k+1} a_{i}\right)}, \quad k \in \mathbb{N}, i \geqq p(k) .
$$

\section{References}

[1] H. Aprola, Characterization of subspaces and quotients of nuclear $L_{f}(\alpha, \infty)$-spaces. Compositio Math. 50, 65-81 (1983).

[2] M. M. Dragilev, On regular bases in nuclear spaces. Amer. Math. Soc. Transl. (2) 93, 61-82 (1970); English translation of Math. Sb. (110) 68, 153-173 (1965). 
[3] J. HebBecker, Auswertung der Splittingbedingungen $S_{1}^{*}$ und $S_{2}^{*}$ ür Potenzreihenräume und $L_{f}$-Räume. Diplomarbeit, Wuppertal 1984.

[4] M. Kocatepe (Alpseymen), On Dragilev spaces and the functor Ext. Arch. Math. 44, 438-445 (1985).

[5] M. KocatePe, Some counterexamples on Dragilev spaces. Doğa Tr. J. Math. (1) 10, 136-142 (1986) (special issue).

[6] J. KRonE-D. VogT, The splitting relation for Köthe-spaces. Math. Z. 180, 387-400 (1985).

[7] D. Vogt, On the functors $\operatorname{Ext}^{1}(E, F)$ for Fréchet spaces. Preprint.

\section{Eingegangen am 16.1.1987}

Anschrift des Autors:

Mefharet Kocatepe

Bilkent University

Faculty of Engineering and Science

Ankara, Turkey 